\title{
Giant Photogalvanic Effect in Noncentrosymmetric Plasmonic Nanoparticles
}

\author{
Sergei V. Zhukovsky, ${ }^{1,2, *}$ Viktoriia E. Babicheva ${ }^{2,1,3}$ Andrey B. Evlyukhin, ${ }^{4}$ Igor E. Protsenko, ${ }^{5,6}$ \\ Andrei V. Lavrinenko, ${ }^{1}$ and Alexander V. Uskov ${ }^{1,5,6}$ \\ ${ }^{1}$ DTU Fotonik-Department of Photonics Engineering, Technical University of Denmark, \\ Ørsteds Plads 343, DK-2800 Kongens Lyngby, Denmark \\ ${ }^{2}$ ITMO University, Kronverksky Prospect 49, Saint Petersburg 197101, Russia \\ ${ }^{3}$ Birck Nanotechnology Center, Purdue University, 1205 West State Street, \\ West Lafayette, Indiana 47907-2057, USA \\ ${ }^{4}$ Laser Zentrum Hannover eingetragener Verein, Hollerithallee 8, D-30419 Hannover, Germany \\ ${ }^{5}$ P. N. Lebedev Physical Institute, Russian Academy of Sciences, \\ Leninskiy Prospect 53, 119333 Moscow, Russia \\ ${ }^{6}$ Advanced Energy Technologies Ltd, Skolkovo, Novaya Ulitsa 100, 143025 Moscow Region, Russia \\ (Received 11 April 2014; revised manuscript received 10 July 2014; published 3 September 2014)
}

Photoelectric properties of noncentrosymmetric, similarly oriented metallic nanoparticles embedded in a homogeneous semiconductor matrix are theoretically studied. Because of the asymmetric shape of the nanoparticle boundary, photoelectron emission acquires a preferred direction, resulting in a photocurrent flow in that direction when nanoparticles are uniformly illuminated by a homogeneous plane wave. This effect is a direct analogy of the photogalvanic (or bulk photovoltaic) effect known to exist in media with noncentrosymmetric crystal structure, such as doped lithium niobate or bismuth ferrite, but is several orders of magnitude stronger. Termed the giant plasmonic photogalvanic effect, the reported phenomenon is valuable for characterizing photoemission and photoconductive properties of plasmonic nanostructures and can find many uses for photodetection and photovoltaic applications.

DOI: 10.1103/PhysRevX.4.031038

\section{INTRODUCTION}

The recent decade in modern physics has featured the concept of optical metamaterials. The central idea of this concept is to bestow the role of known, ordinary constituents of matter (atoms, ions, or molecules) upon artificial "meta-atoms" - nanosized objects purposely designed to have the desired optical properties [1]. The assembly of such meta-atoms - an artificial composite metamaterialexhibits the desired properties macroscopically, provided that the meta-atoms are much smaller than the wavelength of light interacting with them.

As great as the variety of naturally occurring atoms and molecules (and, in turn, of natural materials) may be, the inherent total freedom in choosing the shape and composition of artificial meta-atoms is believed to be even greaternearly arbitrary. Thus, a prominent success of optical metamaterials is the design of materials with optical properties that either do not exist or are much weaker in naturally occurring media. Notable examples include metamaterials with a

\footnotetext{
*sezh@fotonik.dtu.dk
}

Published by the American Physical Society under the terms of the Creative Commons Attribution 3.0 License. Further distribution of this work must maintain attribution to the author(s) and the published article's title, journal citation, and DOI.
Subject Areas: Materials Science,

Metamaterials, Plasmonics negative refractive index, near zero, or very large permittivity [2], metamaterials with magnetic permeability at optical frequencies [3], extremely anisotropic hyperbolic metamaterials that behave like metals in some directions and like dielectrics in others [4-7], chiral metamaterials with giant magnetooptical properties [8-11], and many more.

Most meta-atom designs proposed to date are based on metallic nanoparticles, nanoantennas, or resonators of various shapes [12]. In such metallic structures, the size prerequisite for meta-atom design is fulfilled by subwavelength confinement of electromagnetic field due to localized surface plasmon resonance excitation. At the same time, localized plasmons are known to cause strong local field enhancement, which can enhance the functionality of metamaterials in the context of biological and chemical sensing, as well as give rise to new concepts of optical metamaterials based on strongly enhanced nonlinear, photorefractive, and photoconductive effects. In particular, plasmon-enhanced photoconductivitythe emission of photoelectrons from nanoparticles due to action of strong local fields in the localized plasmonic resonances-was recently shown to be promising for photodetection and photovoltaic applications [13-15].

Transcending the purely electromagnetic approach traditionally adopted in the study of plasmonic nanostructures and accounting for processes when light can cause electrons to leave the nanoparticles has far-reaching implications, 
putting forth a new concept of photoconductive metamaterials. The enhanced photoelectric effect from plasmonic nanoantennas with generation of "hot" electrons $[16,17]$ can be used to improve the characteristics of light-harvesting devices (e.g., photoconductive plasmonic metamaterials, photodetectors, solar and photochemical cells) [16-26], as well as more generally in optoelectronics, photochemistry, and photoelectrochemistry [27-29].

In this paper, we predict and numerically demonstrate an effect related to new functionality of photoconductive metamaterials: the giant plasmonic photogalvanic effect. Named after photogalvanic (or bulk photovoltaic) effect in bulk noncentrosymmetric media [30], plasmonic photogalvanic effect is shown to exist in an array of similarly oriented noncentrosymmetric metallic nanoparticles embedded in a homogeneous semiconductor matrix. The low degree of symmetry of the nanoparticle shape causes the net flux of the hot electrons emitted from the nanoparticles via the resonant plasmonic excitation to be directional. This directionality leads to a photoelectromotive force as a result of homogeneous external light illumination (the photogalvanic effect).

We report that the resulting photocurrent density generated in a layer of nanoparticles emerges and grows as the particle shape changes from cylindrical to conical, i.e., with the increase of the particle asymmetry. We calculate the components of the effective third-rank tensor relating the current density to the incident electric field, and show that this effective tensor for the nanoparticle array exceeds that for the naturally occurring ferroelectrics that exhibit bulk photovoltaic effect. Hence, the reported plasmonic effect can be regarded as a "giant" version of the conventional (nonplasmonic) photogalvanic effect occurring in natural materials.

The paper is organizedas follows. In Sec. II, we review the theoretical background for "hot" electron photoemission at metal-semiconductor interfaces forming Schottky barriers. In Sec. III, we numerically investigate arrays of nanoparticles whose shape varies from cylindrical to conical. We discuss the observed increase of photocurrent directionality and induced electromotive force as a result of increased spatial asymmetry of the nanoparticles. In Sec. IV, we draw parallels between the predicted plasmonic photogalvanic effect and the known photogalvanic effect in certain naturally occurring media. Finally, in Sec. V, we summarize and outline possible applications for the proposed effect.

\section{THEORETICAL BACKGROUND}

We consider a metallic nanoparticle embedded in a uniform semiconductor matrix (Fig. 1) in the presence of a normally incident light wave of frequency $\omega$, such that

$$
W_{b}<\hbar \omega<E_{g} .
$$

(a)
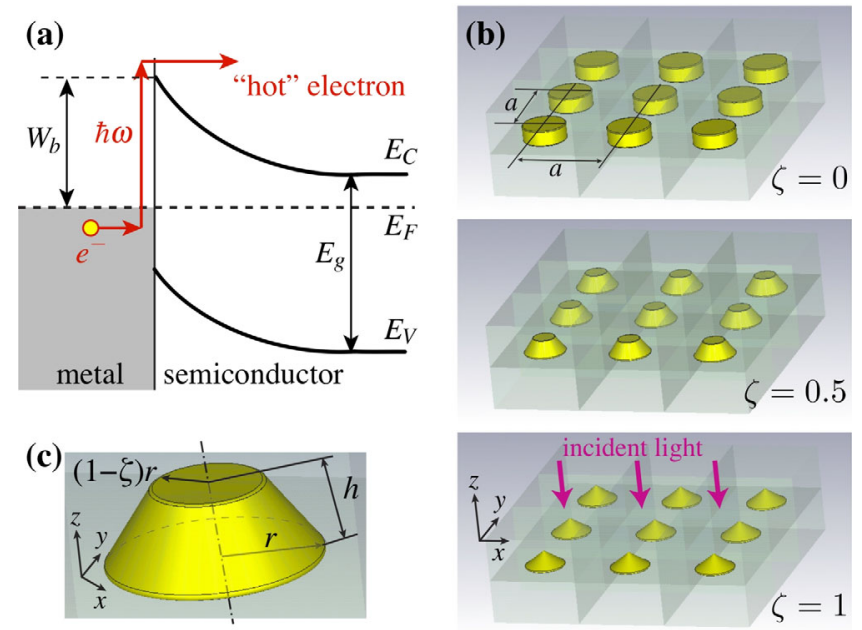

FIG. 1. Schematics of (a) "hot" photoelectron emission through the Schottky barrier at a metal-semiconductor interface; (b) several nanoparticle arrays studied in the present paper (showing three characteristic cases of cylinders, truncated cones, and cones); (c) enlarged view of one nanoparticle showing its geometrical parameters.

It is assumed that the photon energy $\hbar \omega$ is insufficient to excite electron-hole pairs in the semiconductor matrix with gap energy $E_{g}$, but exceeds the work function for the metalsemiconductor interface $W_{b}$ [see Fig. 1(a)], so that the photon energy transferred to an electron in metal can cause it to leave the nanoparticle.

Two mechanisms of energy transfer from the photon to the electron can be identified [31-33]. One is absorption of a photon by an electron in the bulk of the nanoparticle with subsequent transport of the hot electron to the surface and its emission by overcoming the Schottky barrier (the volume photoelectric effect [34]). The other is absorption of a photon by an electron as it collides with the nanoparticle boundary, causing emission of that particular electron from the metal (the surface photoelectric effect). In both of these mechanisms, the spatial and momentum distribution of the emitted electrons is strongly influenced by (i) the spatial configuration of the nanoparticle surface and (ii) resonant field enhancement near that surface due to the plasmonic resonance.

While the question of which of the two mechanisms is stronger in nanostructures is yet to be answered, it was shown that electron collisions after photon absorption leading to rapid hot electron cooldown make surface-driven effects prevail over bulk effects in many cases $[31,35]$. So, following the previously developed Tamm theory of photoemission from plasmonic nanoparticles $[15,24,25]$, we express the photocurrent from a nanoparticle as

$$
I_{\mathrm{NP}}(\lambda)=C_{\mathrm{em}}(\lambda) \oint_{\text {particle }}\left|E_{n}(\mathbf{r})\right|^{2} d^{2} \mathbf{r}
$$


Here, $E_{n}$ is the component of the electric field normal to the surface of the nanoparticle, which is where the integration is performed. The expression in Eq. (2) takes into account only the increased number of photoelectrons without account for the direction of their momentum as they leave the nanoparticle. As a quantity integrated for all electrons in all directions, it is applicable in the photoconductivity scenario when the emitted electrons are subsequently directed using an externally applied or a built-in potential, and their momentum direction upon leaving the nanoparticle can thus be totally disregarded.

In the absence of such potential, the initial velocity of the emitted photoelectrons starts to play a role in the definition of the photocurrent from a nanoparticle, which then has to assume a modified form,

$$
\mathbf{I}_{\mathrm{NP}}(\lambda)=-C_{\mathrm{em}}(\lambda) \oint_{\text {particle }}\left|E_{n}(\mathbf{r})\right|^{2} \mathbf{n} d^{2} \mathbf{r},
$$

where $\mathbf{n}$ is the unit normal vector at the nanoparticle surface at point $\mathbf{r}$. In both Eqs. (2) and (3), the coefficient $C_{\mathrm{em}}$ equals

$$
C_{\mathrm{em}}=\eta_{o} \frac{e}{\hbar \omega} Y_{m}=\eta_{o} \frac{e}{\hbar \omega} \frac{\varepsilon_{0} c n_{m}}{2}
$$

where $n_{m}$ is the refractive index of the matrix and $\eta_{o}$ is the external quantum efficiency of the electron photoemission through the potential barrier at the metal-matrix interface $[15,36]$; it strongly depends on the photon energy and varies from zero at $\hbar \omega=W_{b}$ [see Fig. 1(a)] to nonzero values for higher photon energies [35]. The admittance (inverse impedance) $Y_{m}$ of the matrix medium relates the intensity $S$ of a plane wave with the electric field strength $E$ as $S=Y_{m}|E|^{2}$.

When a plasmonic resonance is excited in the nanoparticle, the amplitude of the resonant fields inside it and near its surface usually greatly exceeds the amplitude of the incident field. Therefore, we see from Eqs. (2) and (3) that in nanoparticles with centrosymmetric shapes (such as nanospheres, nanocubes, or nanodisks) the spatial symmetry of the resonant modes will lead to the cancellation of the directed photocurrent, so that $\mathbf{I}_{\mathrm{NP}}=0$ even though $I_{\mathrm{NP}} \neq 0$. In other words, even though the incident wave can cause additional photoelectrons to be emitted over the Schottky barrier, the collective motion of these electrons will not induce an electromotive force.

The situation changes drastically when the shape of the nanoparticle and/or the field distribution of the resonant plasmonic mode lacks the center of symmetry. Equation (3) then shows that the resulting photocurrent acquires directionality, so that photoemission from such a noncentrosymmetric nanoparticle results in net photocurrent $\left(\mathbf{I}_{\mathrm{NP}} \neq 0\right)$ and induces electromotive force. The ratio $\rho=\left|\mathbf{I}_{\mathrm{NP}}\right| / I_{\mathrm{NP}}$, which can vary from 0 to 1 , can be regarded as a measure of directionality for photoemission with respect to one nanoparticle.

In a planar, oriented arrangement of such noncentrosymmetric nanoparticles, the individual photocurrents from each particle sum up. If the nanoparticles are not too close to each other, the satisfactory approximation is that the neighboring particles do not modify the plasmonic resonance of each other in a significant way. In this case, the resulting photocurrent density from a square nanoparticle lattice with period $a$ can be written as $\mathbf{j}=\mathbf{I}_{\mathrm{NP}} / a^{2}$ with the expression for $\mathbf{I}_{\mathrm{NP}}$ given by Eq. (3) and $E_{n}$ calculated for a single nanoparticle. Since the nanoparticles are axially symmetric with respect to the $z$ axis [see Fig. 1(c)], and the lattice has fourfold rotational symmetry, we expect that for a normally incident wave $j_{x}=j_{y}=0$ and $j_{z}=|\mathbf{j}|$, so we can write

$$
j_{z}=\left|\mathbf{I}_{\mathrm{NP}}\right| / a^{2}=\rho I_{\mathrm{NP}} / a^{2}=\rho C_{\mathrm{em}}\left|E_{0}^{2}\right| \xi,
$$

where $E_{0}$ is the field incident on the lattice, and

$$
\xi=\frac{1}{\left|E_{0}^{2}\right| a^{2}} \oint_{\text {particle }}\left|E_{n}(\mathbf{r})\right|^{2} d^{2} \mathbf{r}
$$

relates the incident field $E_{0}$ with the local field $E(\mathbf{r})$ and has the meaning of a field-enhancement factor due to the localized surface plasmon resonance in the nanoparticles $[15,36]$. If the incident field is, e.g., $x$ polarized, Eq. (5) can be rewritten as

$$
j_{z}=\rho \xi C_{\mathrm{em}} E_{0 x} E_{0 x}^{*}=\tilde{\alpha}_{z x x} E_{0 x} E_{0 x}^{*},
$$

which has the form equivalent to the photocurrent induced in some media with noncentrosymmetric crystal structure due to the photogalvanic (or bulk photovoltaic) effect [37-39],

$$
j_{i}=\alpha_{i j k} E_{j} E_{k}^{*},
$$

where $E_{j}$ are again the components of theincident field, and coefficients $\alpha_{i j k}$, related to the components of a third-rank piezoelectric tensor [37], are nonzero only for noncentrosymmetric media. Examples include piezoelectrics or ferroelectrics such as $\mathrm{LiNbO}_{3}: \mathrm{Fe}$, quartz with $F$ centers, or $p$-GaAs [37]. We see that the photocurrent $\mathbf{j}$ in Eq. (8) is nonlinear (quadratic) with respect to the field strength $E$.

We can also rewrite Eq. (8) in a modified form [40],

$$
j_{i}=\left(\beta_{i j k} / 2\right)\left(e_{j} e_{k}^{*}+e_{j}^{*} e_{k}\right) S_{0},
$$

where $e_{j}=E_{j} /|E|$ is the $j$ th component of the incident light polarization vector, and $S_{0}$ is its intensity. In SI units, the components of the tensor $\beta$ have the dimension of inverse volts; normalized by the absorption coefficients, $\beta_{i j k}$ are related to the Glass coefficients for the high-voltage bulk photovoltaic effect in nonlinear crystals [40,41]. One 
can similarly rewrite Eq. (7), introducing the plasmonic equivalent of Eq. (9), as

$$
j_{z}=\tilde{\beta}_{z x x} S_{0}\left|e_{x}\right|^{2}, \quad \tilde{\beta}_{z x x}=\rho \xi \eta_{o} e /(\hbar \omega) .
$$

We see that Eq. (7) is formally equivalent to Eq. (8), standard for describing the photogalvanic effect in bulk media. However, one important difference has to be noted. In bulk media, directed photoelectrons are generated throughout the volume of the material. They have a finite lifetime since their initial velocity decays as they move. Hence, the coefficients $\alpha_{i j k}$ in Eq. (8) are proportional to that lifetime [30]. In contrast, the geometry in Fig. 1(b) considered here deals with the injection of directed photoelectrons from a nanoparticle array into the surrounding medium. Hence, there is no lifetime in Eqs. (5) and (7), which is emphasized by using a tilde over the coefficients $\tilde{\alpha}_{i j k}$ in that formula.

The behavior of photoelectrons after they have been emitted is a subject for further studies. For one "metalayer" of nanoparticles, the electrons emitted in the direction of the $z$ axis are unlikely to be recaptured by neighboring particles. In this case, photoelectron reabsorption can only be a minor source of losses of emitted electrons, which are not considered in the present study. On the other hand, when many nanoparticle layers are stacked together closely, the emitted photoelectrons can be recaptured and reemitted, and the effective lifetime for the directionally emitted electrons in such 3D metamaterials must be reintroduced.

\section{DIRECTIONAL PHOTOEMISSION FROM CONICAL NANOPARTICLES}

To confirm the predicted photogalvanic effect in a plasmonic structure, we consider an array of gold nanoparticles whose shape is gradually varied from cylindrical to conical [Figs. 1(b) and 1(c)]. The choice of conical nanoparticles is well motivated from a fabricational standpoint, since nanodisks fabricated using lithographic means often acquire asymmetry and resemble conelike shapes $[42,43]$; other asymmetric shapes, such as hemispheres and nanopyramids, are also readily fabricable using various techniques [44-46].

Let the nanocones under study have height $h$; the larger (bottom) facet has radius $r$, and the radius of the smaller (top) facet is given by $r(1-\zeta)$, so that $\zeta$ can be understood as the "asymmetry" or "conicity" parameter. The case $\zeta=$ 0 corresponds to the centrosymmetric, disk-shaped particles, whereas the opposite case $\zeta=1$ corresponds to maximally asymmetric nanocones. For the computational example, we use $r=25 \mathrm{~nm}$ and $h=18 \mathrm{~nm}$.

The particles are embedded in a homogeneous GaAs matrix $\left(n_{m}=3.6, W_{b}=0.8 \mathrm{eV}, E_{g}=1.43 \mathrm{eV}\right)$, which results in the operating range between 870 and $1550 \mathrm{~nm}$ according to Eq. (1). For the $\mathrm{Au} / \mathrm{GaAs}$ interface, the values of $\eta_{o}$ range from zero at $\hbar \omega=W_{b}$ to about 0.0025 for $\hbar \omega \lesssim$ $E_{g}$ [35]. Because of such a small probability for the photons' energy to be transferred to hot electrons, we can assume that their formation does not significantly add to the Ohmic losses in metal. Therefore, we can use the Drude model of the gold permittivity, known to be a good fit for photon energies up to $2 \mathrm{eV}$ [47], with plasma frequency $2.18 \times$ $10^{15} \mathrm{~s}^{-1}$ and collision frequency $6.47 \times 10^{12} \mathrm{~s}^{-1}$ [48].

The particles are arranged in a $2 \mathrm{D}$ square lattice with period $a=100 \mathrm{~nm}$. According to earlier results [15,25], such a dense lattice prevents the appearance of higher-order diffraction and ensures that the lattice effects are outside of the operating range, so the resonant mode of a particle in a lattice largely coincides with that of an isolated particle [15]. Another benefit of making the lattice dense is the increase of the nanoparticles' concentration, resulting in the increase of the total induced photocurrent as per Eq. (5).

Simulations were carried out in the frequency domain using CST MICROWAVE STUDIO. The results showthat all of the structures in question feature a rather broad absorption resonance corresponding to the excitation of a localized surface plasmon [Fig. 2(a)]. As expected, small $a$ makes the lattice-related resonances occur outside of the operating frequency range, so the resonance is dominated by the response of a single plasmonic nanoparticle, broadened due to the presence of many nanoparticles [15]. Additional resonances typically present in triangular particles [49] are not excited because light is incident from the direction coincident with the axis of the cones. Note that, to reduce the influence of the discretization ("staircasing") artifacts at sharp edges (which would anyway be unphysical from the fabricational point of view), all edges of the nanoparticles were smoothed with the curvature radius of $\delta r=1 \mathrm{~nm}$. Together with the adaptive mesh refinement, this proved successful in eliminating numerical artifacts from meshingrelated "hot spots."

We further see that the resonance strongly depends on the nanoparticle shape. As the shape changes from a cylinder to a cone, the resonance undergoes a very slight blueshift up to $\zeta=0.4$; further increase of $\zeta$ leads to a strong redshift accompanied by a rather significant narrowing of the resonance. Plotting the normal component of the resonant mode field on the nanoparticle surface [Fig. 2(b)], we notice that although the field at the smaller base (or tip) of the cone becomes progressively weaker as $\zeta$ increases, the mode maintains the characteristic outside field pattern of a fundamental dipole resonance for all nanoparticles. To reconfirm this, we calculate the extinction cross section of conical nanoparticles using the discrete dipole approximation (DDA) method [50,51]. Figure 2(c) shows that extinction properties of the nanoparticles are fully reproduced if only the electric dipole moment is kept in the cases of both low and high asymmetry.

The DDA results reproduce the redshift of the resonance as $\zeta$ increases, which is qualitatively similar to what 
(a)

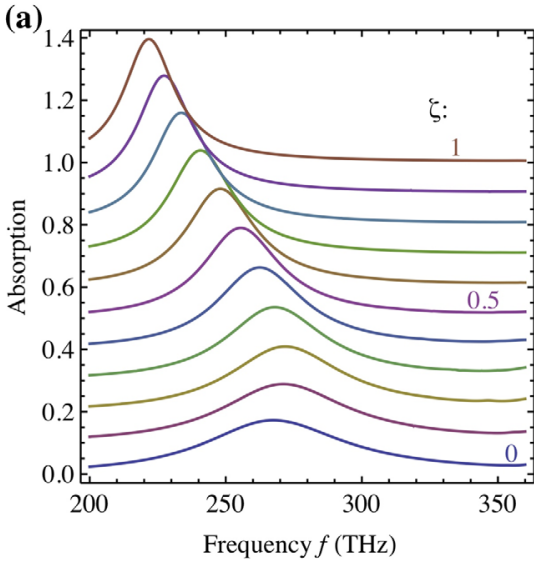

(b)
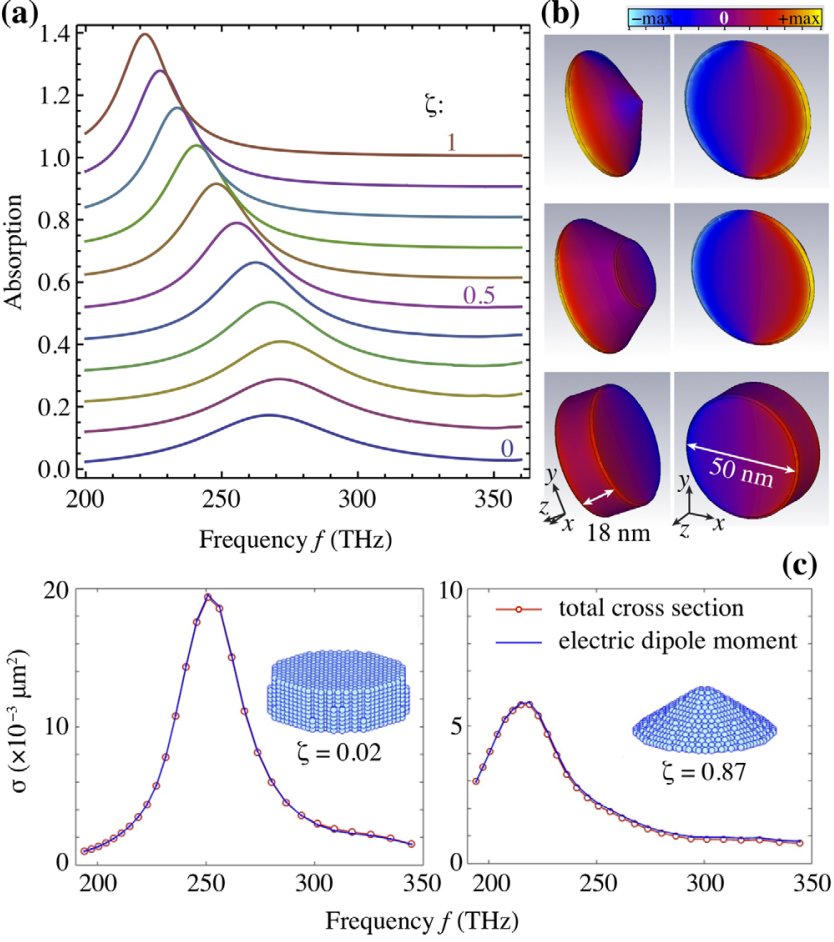

FIG. 2. (a) Absorption spectra for the nanoparticle lattice with the conicity parameter $\zeta$ ranging from 0 (cylinders) to 1 (cones). The light is normally incident onto the lattice plane and linearly polarized along the $x$ axis. For easier readability, each line is offset by 0.1. (b) Images of the normal component of the electric field at the plasmonic resonance (the frequency of the absorption maximum) for $\zeta=0,0.5$, and 1 , viewed from two different angles.(c) Extinction cross section for a single nanoparticle with near-cylindrical $(\zeta=0.02)$ and highly conical $(\zeta=0.87)$ shape, calculated with the DDA method comparing the full cross section (dots) with the electric dipole moment contribution (blue line); the insets show the images of the discretized nanoparticles.

happens in a metal spheroid as it becomes more oblate [52]. Moreover, the results in Fig. 2(c) show the decrease of the effective dipole moment of a nanocone compared to a nanodisk. This decrease diminishes the coupling between the individual particles in the lattice, weakening the absorption peak broadening for larger $\zeta$, which is thought to be the primary mechanism of the resonance narrowing for the nanocones.

From the distribution of the local field at the nanoparticle surface, which is determined numerically at a set of frequencies throughout the operating range, we then calculate the photoemission current from each nanoparticle using Eqs. (2) and (3). The results are shown in Figs. 3(a) and $3(\mathrm{~b})$, respectively. We see that the field enhancement factor $\xi$ has the maximum, which coincides in frequency with the absorption resonance in Fig. 2(a), causing the maximum in both the total and the directional photocurrent. More importantly, we see that the cylindrical nanoparticles have no preferred direction of the emitted photoelectrons. However, the more the nanoparticle shape evolves towards
Total photocurrent, $I_{\mathrm{NP}} \sim \xi \quad$ Directional photocurrent, $\left|\mathbf{I}_{\mathrm{NP}}\right| \sim \rho \xi$

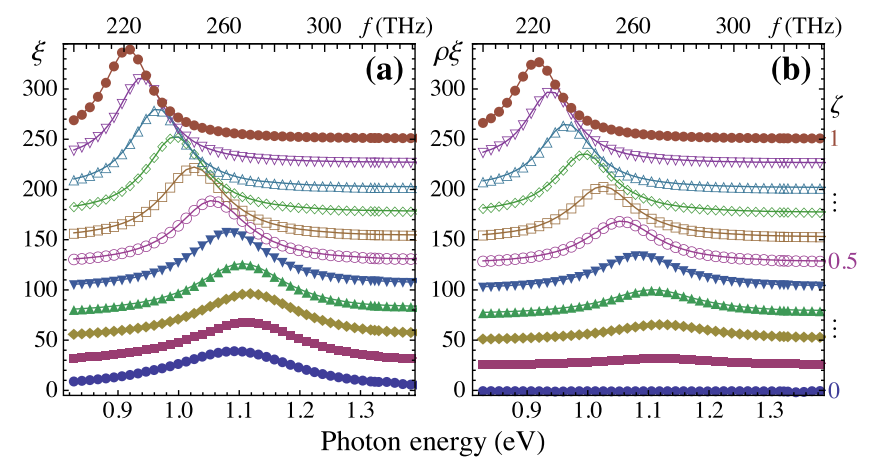

FIG. 3. (a) Total photocurrent $I_{\mathrm{NP}}$ and (b) $z$ component of the directional photocurrent $\mathbf{I}_{\mathrm{NP}}$ for different values of the conicity parameter $\zeta$. The lines are offset by 25 for easier readability.

conical with $z$-axis symmetry, the greater directionality along the $z$ axis the photoelectrons acquire.

The sign of $\mathbf{j}_{z}$ indicates that electrons tend to be emitted in the direction of the base of the cone. This can be explained by the field distributions in Fig. 2(b). In a nanodisk, the two facets have equal field distribution and therefore the photocurrent resulting from emission from the two facets balances each other; the same happens with the sidewalls, resulting in overall $\mathbf{I}_{\mathrm{NP}}=0$. In a cone, the field distribution (and hence, photoemission) at its base is similar to the facet of the nanodisk, but the field at the remaining surface of the cone is significantly weaker compared to the base because the center of mass of a nanocone gets shifted towards its base as its asymmetry increases, again qualitatively similar to an oblate metal spheroid [52]. Therefore, as $\zeta$ increases, the smaller facet of the cone has a gradually declining contribution to the total photoemission process, thus increasing the photocurrent directionality.

Since the operating frequency range contains only one plasmonic resonance, the increase in $\rho$ is almost uniform across the spectrum. We can thus introduce an overall spectrally averaged $\bar{\rho}$ for the structure with a given $\zeta$; the resulting dependence $\bar{\rho}(\zeta)$ is shown in Fig. 4. We see that highly asymmetric nanoparticles $(\zeta>0.5)$ display significant $\rho$ that exceeds 0.5 , while for fully conical nanoparticles, $\rho>0.8$.

Moreover, we see that the field enhancement $\xi$ also becomes greater when particles become more asymmetric, with maximum $\xi$ changing from about 40 for cylinders to almost 100 for cones. Using Eq. (10) and the expression for $\eta_{o}$ for the $\mathrm{Au} / \mathrm{GaAs}$ interface calculated in Ref. [35] at the maximum of the dependence $\xi(\hbar \omega)$ [see Fig. 3(a)], we can finally derive the effective tensor component $\tilde{\beta}_{z x x}$, also shown in Fig. 4. We see that the maximum value of around 0.07 is reached for conicity parameter values $\zeta \simeq 0.6-0.7$ (Fig. 4). For more asymmetric shapes, the increase in $\xi$ and $\rho$ is compensated by the shift of plasmonic resonance towards $\hbar \omega=W_{b}$, where $\eta_{o}$ rapidly approaches zero, 


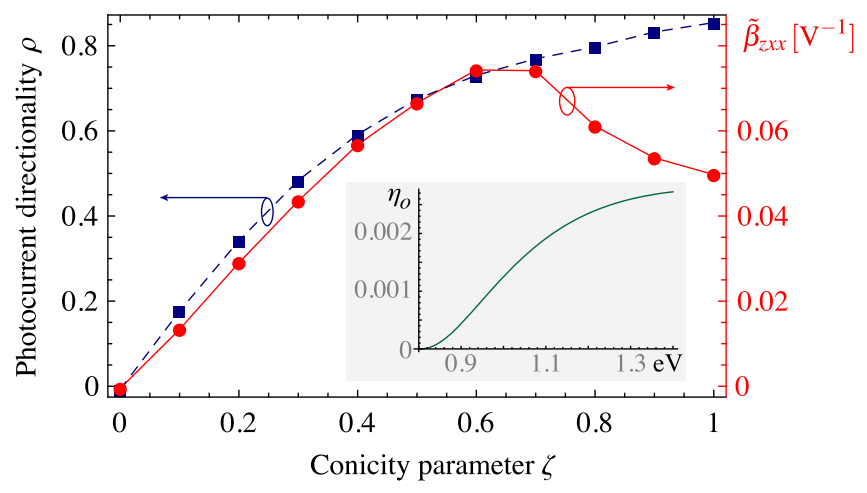

FIG. 4. Photocurrent directionality ratio $\bar{\rho}$ averaged for the photon energies between 1.0 and $1.1 \mathrm{eV}$ (squares) and calculated value of the effective $\tilde{\beta}_{z x x}$ (circles) for the value of $\eta_{o}$ for the photon energy of maximum photocurrent [the peak in Fig. 3(a)] calculated according to Ref. [35] (also shown in the inset), depending on the conicity parameter $\zeta$.

leading to a slight decrease in $\tilde{\beta}_{z x x}$ to values around 0.05 . Changing the nanoparticle's aspect ratio in such a way that its plasmonic resonance gets shifted to higher frequencies (exceeding $1 \mathrm{eV}$ ) is expected to counteract this effect and further boost the directional photocurrent.

The resulting values of $\tilde{\beta}_{z x x}$ in Fig. 4 are seen to greatly exceed the typical values for ferroelectric crystals, such as the experimentally determined $\beta_{x x x}=3.1 \times 10^{-12} \mathrm{~V}^{-1}$ in $\mathrm{La}_{3} \mathrm{Ga}_{5} \mathrm{SiO}_{14}: \mathrm{Fe}$ [40]. The obtained $\tilde{\beta}_{z x x}$ is also found to exceed the anomalously high values for bismuth ferrite known to outperform typical ferroelectric materials by about 5 orders of magnitude in the thin-film configuration $\left[\beta_{i j j}\right.$ around $(2-3) \times 10^{-4} \mathrm{~V}^{-1}$ according to the recent measurements [53] and first-principles theoretical calculations [39]]. Furthermore, it exceeds the values $\beta_{i j j} \sim$ $10^{-4}-10^{-3} \mathrm{~V}^{-1}$ calculated for the photogalvanic effect based on ratchet photocurrent from interaction of free electrons with asymmetric nanoscatterers without taking any plasmonic effects into consideration [38].

Even though the calculations are made for a relatively dense array of nanoparticles, Eq. (6) gives a rough estimate of how $\tilde{\beta}_{z x x}$ would change with $a$ : increasing it by 3 times lowers $\tilde{\beta}_{z x x}$ by a factor of 9 . A more precise analysis involves actual calculation of the modified field distribution at the plasmonic resonance in sparser arrays. In accordance with earlier predictions for plasmonic nanodisks [15], calculations show that the local field enhancement gets stronger in sparser arrays, which additionally increases $\tilde{\beta}_{z x x}$. As a result, the predicted plasmonic photogalvanic effect persists for nanoparticle arrays with $a$ up to $300 \mathrm{~nm}$, above which the array starts to resemble a diffraction grating rather than a subwavelength metalayer. Likewise, the effective values of $\tilde{\beta}_{z x x}$ are found to decrease by about 1 order of magnitude if the damping factor of the metal increases by 3 times, which makes it likely for the predicted effect to be still observable in lossier metals.
On the other hand, we stress that the structure example used here is not subject to any optimization specifically directed at maximizing $\tilde{\beta}_{z x x}$. We expect that by optimizing the nanocones' shape in such a way that their plasmonic resonance gets narrower (which increases $\xi$ ) and moves towards higher frequencies (which increases $\eta_{o}$ ) the plasmonic photogalvanic effect can be made even stronger. These arguments, combined with the estimations made above, permit us to conclude that the predicted photogalvanic tensor values do not hinge on a specific choice of geometric or material parameters, and are indeed indicative of the platform. Hence, the plasmonic photogalvanic effect in oriented arrangements of asymmetrically shaped nanoparticles can be confirmed to constitute a "giant" version of bulk photovoltaic effect present in noncentrosymmetric crystals.

\section{PLASMONIC VERSUS CONVENTIONAL PHOTOGALVANIC EFFECT}

Having established formal equivalence between the conventional bulk photovoltaic effect in noncentrosymmetric crystalline materials [37] and the reported plasmonic effect in nanoparticles through the parallels between Eqs. (7) and (8), or similarly between Eqs. (9) and (10), we now discuss the similarities in the underlying physics behind these two effects in more detail.

Conventional photogalvanic effect [54] or bulk photovoltaic effect [37] (sometimes called high-voltage bulk photovoltaic effect [55]) refers to the generation of intrinsic photocurrents occurring in single-phase materials without inversion symmetry [56]. Microscopically, it is associated with violation of the principle of detailed balance for photoexcited nonequilibrium carriers in noncentrosymmetric crystals: the probability of the electron transition between the states with momentum $k$ and $k^{\prime}, W\left(k, k^{\prime}\right)$, does not, in general, equal the probability of the reverse transition: $W\left(k, k^{\prime}\right) \neq W\left(k,-k^{\prime}\right)$ [57]. This gives rise to a flux of photoexcited carriers, which manifests itself as photocurrent with a certain direction even though the medium is homogeneous and uniformly illuminated.

The principle of detailed balance may be violated due to a variety of mechanisms, e.g., inelastic scattering of carriers on noncentrosymmetric centers, excitation of impurity centers with an asymmetric potential, or a hopping mechanism that acts between asymmetrically distributed centers [37]. Other effects that have been pointed out are excitation of nonthermalized electrons having asymmetric momentum distribution due to crystal asymmetry, delocalized optical transitions in lattice excitation of pyroelectrics [55], spin-orbital splitting of the valence band in gyrotropic media [37], and second-order nonlinear optical interaction known as "shift currents," shown to be the dominating photocurrent cause in ferroelectrics [39,56].

The latter effect is strikingly similar to the plasmonic effect reported here because the expression for the shift 
current, $J_{q}=\sigma_{r s q} E_{r} E_{s}$, is essentially coincident with Eqs. (7) and (8); in both cases, the photocurrent is quadratic with respect to the field strength of incident light [24].

Another striking similarity arises when one compares the considered photoemission from nanoparticles with photoionization from atoms. Indeed, one can regard nanoparticles as atoms whose electrons are placed into a highly asymmetric potential well, which is formed by the boundary of metal with the surrounding medium. Then, it is known from atomic physics that the pattern of photoeffect from such atoms would depend on the shape of the atomic potential well. From this point of view, it is clear that changing the shape of the nanoparticles can efficiently deform the pattern of electron photoemission from such nanoparticles in much the same way as what happens in noncentrosymmetric crystals where asymmetry is inherent in the crystal lattice structure.

Hence, we have solid ground to regard the reported plasmonic effect in nanoparticle arrangements as the plasmonic analogy to the conventional photogalvanic or bulk photovoltaic effect, if we think of nanoparticles as meta-atoms and equate the absence of the center of symmetry in them to a similar geometric feature of crystal lattice in bulk media. In some ways, it resembles the mesoscopic photovoltaic effect that was reported earlier to occur in ensembles of semiconductor microjunctions of larger dimensions (about $1 \mu \mathrm{m}$ ) in the microwave range $[58,59]$.

Choosing between the terms photogalvanic and bulk photovoltaic to name the reported effect is worth another discussion. In homogeneous media, these two terms are essentially synonymous and have been used interchangeably. Indeed, historically the words "galvanic" and "voltaic," attributed to Galvani and Volta, respectively (both of whom were behind the invention of a battery), have the same meaning. In later use, though, the term photovoltaic effect gained much wider recognition and at the same time became much more generic; it came to mean any effect of electric energy generation as a result of light illumination (perhaps with the exception of photoelectron emission into vacuum, for which the term photoelectric effect remains more popular). Still, the predominant usage realm of photovoltaic effects became that of the processes in modern-day semiconductor solar cells, i.e., the effects related to generation and subsequent separation of electrons and holes in semiconductor structures. To distinguish these heterostructure effects from photocurrent generation in the bulk of a noncentrosymmetric homogeneous medium, the latter adopted the name "bulk photovoltaic effect"; its much less popular synonym "photogalvanic effect" has not needed this addition.

For this reason, in our attempt to classify the predicted plasmonic effect, adopting the name plasmonic bulk photovoltaic effect can be confusing because one is tempted to forget that the plasmonic structure under study is only "effectively bulk" in the sense that its characteristic dimensions are much smaller than the wavelength of the incident light. If this subwavelength condition is fulfilled with respect to the size of the nanocones and the distance between them, i.e., if $\lambda / n_{m} \gg \max (2 r, h)$ and $\lambda / n_{m}>a$, then light will interact with the nanocone array as if it were a homogeneous slab of an effective metamaterial. Microscopically, though, it is not homogeneous; the very existence of localized surface plasmon excitations implies that there are surfaces that give rise to them. The wording "bulk plasmonic photovoltaic effect" would be even more dangerous because it may mislead one into thinking that bulk plasmons, rather than surface plasmons, are at work, which is not the case.

Therefore, to avoid such confusions, we argue that plasmonic photogalvanic effect is the proper name for the reported phenomenon. In the broader picture, it has attributes of both a bulk effect and a surface effect depending on the length scale, and in this way, it bridges the gap between the inner photoelectric effect (defined as electric charge carrier generation due to photon absorption in a bulk material) and the outer photoelectric effect (defined as electric charge carrier emission from one medium into another across an interface) [60]; this is not to be confused with internal versus external photoelectric effect, both of which are subclasses of the outer photoelectric effect depending on whether the second medium is solid or not [61].

One does need to reiterate, however, that even when the above-mentioned subwavelength considerations are fulfilled, the present numerical demonstration of plasmonic photogalvanic effect, based on a 2D arrangement of nanoparticles, has only succeeded in demonstrating the equivalence for a "thin slab" of metamaterial, analogous to a thin film of a bulk noncentrosymmetric crystalline medium. Further comparison of plasmonic and conventional photogalvanic effect involving a 3D arrangement of nanoparticles should, therefore, be forthcoming. It is rather straightforward, with the two key challenges being (i) the means to provide uniform illumination in a medium with sufficiently many lossy metallic inclusions under the condition of localized surface plasmon resonance (and hence, highly inhomogeneous and strongly enhanced local fields) and (ii) the possibility for photoelectrons emitted from one nanoparticle to be reabsorbed by other nanoparticles if their density is sufficiently high. Overcoming these challenges may result in a cap on the maximum nanoparticle density and, therefore, a limit on how strong the plasmonic photogalvanic effect can be in 3D arrangements.

That said, the results presented in the numerical demonstration in Sec. III point out that, in terms of the relevant tensor components, plasmonic photogalvanic effect can be several orders of magnitude stronger than the conventional one. Hence, calling the effect "giant plasmonic photogalvanic effect" is warranted, on par with 
giant magnetooptical effects present in chiral metamaterials and similarly surpassing the naturally occurring chirality in bulk media by orders of magnitude [8].

\section{CONCLUSIONS AND OUTLOOK}

To summarize, we theoretically predict new functionality in photoconductive metamaterials: the giant plasmonic photogalvanic effect, analogous to the photogalvanic (or bulk photovoltaic) effect in homogeneous noncentrosymmetric media [30]. The reported effect is numerically demonstrated in an array of similarly oriented noncentrosymmetric metallic nanoparticles embedded in a homogeneous semiconductor matrix, when illuminated by a wave with photon energies insufficient for the internal photoelectric excitation in the semiconductor. Because of the lower degree of symmetry in the nanoparticles (the absence of mirror symmetry with respect to the $x-y$ plane), the flux of "hot" electrons emitted from the nanoparticles with the assistance of a resonant plasmonic excitation acquires directionality along the $z$ axis. Averaged over the volume of the metalayer, this directionality is manifest as an electromotive force resulting from homogeneous external light illumination (the photogalvanic effect).

We also find that the resulting current density generated in a layer of nanoparticles grows as the particle shape changes from cylindrical to conical. Furthermore, we calculate the component $\tilde{\beta}_{z x x}$ of the effective third-rank tensor that relates the induced current density to the incident electric field intensity [see Eq. (10)]. We show that the effective $\tilde{\beta}_{z x x}$ for the nanoparticle array exceeds the components $\beta_{i j k}$ for the naturally occurring ferroelectrics with bulk photovoltaic effect $[39,40,53]$ by orders of magnitude. Hence, the reported plasmonic effect can be regarded as a "giant" version of the photogalvanic effect occurring in natural materials, adding to the assortment of effects that are much stronger in artificial metamaterials than in natural media.

On a fundamental level, the proposed effect is important for our understanding of plasmon-assisted electron photoemission processes, and constitutes a new way of exploring light-matter interaction at the mesoscopic scale. On a more applied level, our results can be used in a variety of ways, from a new way of characterizing plasmonic structures (distinct from purely optical or electron-microscopy approaches) to new designs of photodetectors able to operate outside of the spectral range for band-to-band transitions for semiconductors. It can also be used to increase the performance of photovoltaic elements by making use of longer-wavelength photons, which are normally lost in traditional cells based on the inner photoelectric effect $[22,25]$. The result that photoemission predominantly occurs at the base of the nanocones makes them particularly appealing for photovoltaic devices where nanoparticles are deposited on a semiconductor substrate.
Moreover, we can regard photoemission in noncentrosymmetric plasmonic nanoantennas as a "ratchet" (Brownian-motor) mechanism $[62,63]$ that works as an optical rectifier or "rectenna" [64]. The fundamental concept of optical ratchet devices and optical rectennas attracts much attention in recent developments in nanotechnology [65-68].

Finally, we note that we consider only the plasmonic analog of the linear bulk photovoltaic effect, since the nanoparticle shape is chosen to be achiral. It is expected that chiral (or planar chiral) nanoparticles would provide the plasmonic analog to the circular bulk photovoltaic effect $[30,40]$, described in analogy with Eq. (9) as

$$
j_{i}^{C}=i \beta_{i j}^{C}\left[\mathbf{e} \times \mathbf{e}^{*}\right]_{j} S_{0} .
$$

Thus, designing plasmonic nanostructures with anomalously high effective $\tilde{\beta}_{i j}^{C}$ is expected to result in new ways to characterize both chiral plasmonic nanostructures and chirality-related properties of light.

\section{ACKNOWLEDGMENTS}

We thank Jesper Mørk for valuable comments. S. V.Z. acknowledges support from the People Programme (Marie Curie Actions) of the European Union's 7th Framework Programme FP7-PEOPLE-2011-IIF under REA Grant Agreement No. 302009 (Project HyPHONE). V.E. B. acknowledges support from SPIE Optics and Photonics Education Scholarship, as well as Otto Mønsteds and Kaj og Hermilla Ostenfeld foundations. I. E. P. and A. V. U. acknowledge support from the Russian MSE State Contract No. N14.527.11.0002 and from Russian Foundation for Basic Research (RFBR).

[1] W. Cai and V.M. Shalaev, Optical Metamaterials: Fundamentals and Applications (Springer, Berlin, 2009).

[2] V. M. Shalaev, Optical Negative-Index Metamaterials, Nat. Photonics 1, 41 (2007).

[3] W. Cai, U. K. Chettiar, H.-K. Yuan, V. C. de Silva, A. V. Kildishev, V. P. Drachev, and V. M. Shalaev, Metamagnetics with Rainbow Colors, Opt. Express 15, 3333 (2007).

[4] Y. Liu, G. Bartal, and X. Zhang, All-Angle Negative Refraction and Imaging in a Bulk Medium Made of Metallic Nanowires in the Visible Region, Opt. Express 16, 15439 (2008).

[5] S. Foteinopoulou, M. Kafesaki, E. N. Economou, and C. M. Soukoulis, Two-Dimensional Polaritonic Photonic Crystals as Terahertz Uniaxial Metamaterials, Phys. Rev. B 84, 035128 (2011).

[6] A. Poddubny, I. Iorsh, P. Belov, and Yu. Kivshar, Hyperbolic Metamaterials, Nat. Photonics 7, 948 (2013).

[7] G. V. Naik, B. Saha, J. Liu, S. M. Saber, E. A. Stach, J. M. K. Irudayaraj, T. D. Sands, V. M. Shalaev, and A. Boltasseva, Epitaxial Superlattices with Titanium Nitride as a Plasmonic 
Component for Optical Hyperbolic Metamaterials, Proc. Natl. Acad. Sci. U.S.A. 111, 7546 (2014).

[8] M. Kuwata-Gonokami, N. Saito, Y. Ino, M. Kauranen, K. Jefimovs, T. Vallius, J. Turunen, and Y. Svirko, Giant Optical Activity in Quasi-Two-Dimensional Planar Nanostructures, Phys. Rev. Lett. 95, 227401 (2005).

[9] M. Decker, M. W. Klein, M. Wegener, and S. Linden, Circular Dichroism of Planar Chiral Magnetic Metamaterials, Opt. Lett. 32, 856 (2007).

[10] R. Singh, E. Plum, C. Menzel, C. Rockstuhl, A. K. Azad, R. A. Cheville, F. Lederer, W. Zhang, and N. I. Zheludev, Terahertz Metamaterial with Asymmetric Transmission, Phys. Rev. B 80, 153104 (2009).

[11] Z. Li, Chiral Metamaterials: From Optical Activity and Negative Refractive Index to Asymmetric Transmission, J. Opt. 15, 023001 (2013).

[12] P. Biagioni, J. Huang, and B. Hecht, Nanoantennas for Visible and Infrared Radiation, Rep. Prog. Phys. 75, 024402 (2012).

[13] M. W. Knight, H. Sobhani, P. Nordlander, and N. J. Halas, Photodetection with Active Optical Antennas, Science 332, 702 (2011).

[14] A. Sobhani, M. W. Knight, Y. Wang, B. Zheng, N. S. King, L. V. Brown, Z. Fang, P. Nordlander, and N. J. Halas, Narrowband Photodetection in the Near-Infrared with a Plasmon-Induced Hot Electron Device, Nat. Commun. 4, 1643 (2013).

[15] S. V. Zhukovsky, V. E. Babicheva, A. V. Uskov, I. E. Protsenko, and A. V. Lavrinenko, Enhanced Electron Photoemission by Collective Lattice Resonances in Plasmonic Nanoparticle-Array Photodetectors and Solar Cells, Plasmonics 9, 283 (2014).

[16] A. O. Govorov, H. Zhang, and Y. K. Gun'ko, Theory of Photoinjection of Hot Plasmonic Carriers from Metal Nanostructures into Semiconductors and Surface Molecules, J. Phys. Chem. 117, 16616 (2013).

[17] H. Chalabi and M. Brongersma, Plasmonics: Harvest Season for Hot Electrons, Nat. Nanotechnol. 8, 229 (2013).

[18] Y. Nishijima, K. Ueno, Y. Yokota, Kei Murakoshi, and H. Misawa, Plasmon-Assisted Photocurrent Generation from Visible to Near-Infrared Wavelength Using a Au-Nanorods $/ \mathrm{TiO}_{2}$ Electrode, J. Phys. Chem. Lett. 1, 2031 (2010).

[19] Y. Takahashi and T. Tatsuma, Solid State Photovoltaic Cells Based on Localized Surface Plasmon-Induced Charge Separation, Appl. Phys. Lett. 99, 182110 (2011).

[20] M. W. Knight, Y. Wang, A. S. Urban, A. Sobhani, B. Y. Zheng, P. Nordlander, and N. J. Halas, Embedding Plasmonic Nanostructure Diodes Enhances Hot Electron Emission, Nano Lett. 13, 1687 (2013).

[21] E. Moulin, U. Paetzold, B. Pieters, W. Reetz, and R. Carius, Plasmon-Induced Photoexcitation of "Hot" Electrons and "Hot" Holes in Amorphous Silicon Photosensitive Devices Containing Silver Nanoparticles, J. Appl. Phys. 113, 144501 (2013).

[22] T. P. White and K. R. Catchpole, Plasmon-Enhanced Internal Photoemission for Photovoltaics: Theoretical Efficiency Limits, Appl. Phys. Lett. 101, 073905 (2012).

[23] S. Mubeen, J. Lee, N. Singh, S. Krämer, G. D. Stucky, and M. Moskovits, An Autonomous Photosynthetic Device in
Which All Charge Carriers Derive from Surface Plasmons, Nat. Nanotechnol. 8, 247 (2013).

[24] I. E. Protsenko and A. V. Uskov, Photoemission from Metal Nanoparticles, Phys. Usp. 55, 508 (2012).

[25] A. Novitsky, A. V. Uskov, C. Gritti, I. E. Protsenko, B. E. Kardynał, and A. V. Lavrinenko, Photon Absorption and Photocurrent in Solar Cells below Semiconductor Bandgap due to Electron Photoemission from Plasmonic Nanoantennas, Prog. Photovoltaics 22, 422 (2014).

[26] A. J. Leenheer, P. Narang, N. S. Lewis, and H. A. Atwater, Solar Energy Conversion via Hot Electron Internal Photoemission in Metallic Nanostructures: Efficiency Estimates, J. Appl. Phys. 115, 134301 (2014).

[27] M. Sun and H. Xu, A Novel Application of Plasmonics: Plasmon-Driven Surface-Catalyzed Reactions, Small 8, 2777 (2012).

[28] Y. Liu, H. Zhai, F. Guo, N. Huang, W. Sun, C. Bu, T. Peng, J. Yuan, and X. Zhao, Synergistic Effect of Surface Plasmon Resonance and Constructed Hierarchical $\mathrm{TiO}_{2}$ Spheres for Dye-Sensitized Solar Cells, Nanoscale 4, 6863 (2012).

[29] N. Liu, H. Wei, J. Li, Z. Wang, X. Tian, A. Pan, and H. Xu, Plasmonic Amplification with Ultra-High Optical Gain at Room Temperature, Sci. Rep. 3, 1967 (2013).

[30] B. I. Sturman and V. M. Fridkin, The Photovoltaic and Photorefractive Effects in Noncentrosymmetric Materials (Gordon and Breach, Philadelphia, 1992).

[31] I. Tamm and S. Schubin, Zur Theorie des Photoeffektes an Metallen, Z. Phys. 68, 97 (1931).

[32] A. M. Brodsky and Y. Y. Gurevich, Theory of Electron Emission from Metals (Nauka, Moscow, 1973).

[33] A. M. Brodskii and Yu. Ya. Gurevich, Theory of External Photoeffect from the Surface of a Metal, J. Exp. Theor. Phys. 27, 114 (1968).

[34] C. Scales and P. Berini, Thin-Film Schottky Barrier Photodetector Models, IEEE J. Quantum Electron. 46, 633 (2010).

[35] A. V. Uskov, I. E. Protsenko, R. Sh. Ikhsanov, V.E. Babicheva, S. V. Zhukovsky, A. V. Lavrinenko, E. P. O'Reilly, and H. Xu, Internal Photoemission from Plasmonic Nanoparticles: Comparison between Surface and Volume Photoelectric Effects, Nanoscale 6, 4716 (2014).

[36] S. V. Zhukovsky, V. E. Babicheva, A. V. Uskov, I. E. Protsenko, and A. V. Lavrinenko, Electron Photoemission in Plasmonic Nanoparticle Arrays: Analysis of Collective Resonances and Embedding Effects, Appl. Phys. A 116, 929 (2014).

[37] V. M. Fridkin, Bulk Photovoltaic Effect in Noncentrosymmetric Crystals, Crystallogr. Rep. (Transl. Kristallografiya) 46, 654 (2001).

[38] A. D. Chepelianskii, M. V. Entin, L. I. Magarill, and D. L. Shepelyansky, Theory of Photogalvanic Effect in Asymmetric Nanostructure Arrays, Physica (Amsterdam) 40E, 1264 (2008).

[39] S. M. Young, F. Zheng, and A. M. Rappe, First-Principles Calculation of the Bulk Photovoltaic Effect in Bismuth Ferrite, Phys. Rev. Lett. 109, 236601 (2012).

[40] T. M. Batirov, K. A. Verkhovskaya, R. K. Dzhalalov, E. V. Dubovik, B. V. Mill, and V. M. Fridkin, Bulk Photovoltaic and Photorefractive Effects in a Piezoelectric 
$\mathrm{La}_{3} \mathrm{Ga}_{5} \mathrm{SiO}_{14}$ :Fe crystal, Crystallogr. Rep. (Transl. Kristallografiya) 45, 154 (2000).

[41] A. M. Glass, D. von der Linde, and T. J. Negran, HighVoltage Bulk Photovoltaic Effect and the Photorefractive Process in $\mathrm{LiNbO}_{3}$, Appl. Phys. Lett. 25, 233 (1974).

[42] V. Ferry, J. Munday, and H. Atwater, Design Considerations for Plasmonic Photovoltaics, Adv. Mater. 22, 4794 (2010).

[43] H. A. Atwater and A. Polman, Plasmonics for Improved Photovoltaic Devices, Nat. Mater. 9, 205 (2010).

[44] K. Nakayama, K. Tanabe, and H. A. Atwater, Plasmonic Nanoparticle Enhanced Light Absorption in GaAs Solar Cells, Appl. Phys. Lett. 93, 121904 (2008).

[45] A. Kirkeminde, M. Retsch, Q. Wang, G. Xu, R. Hui, J. Wud, and S. Ren, Surface-Passivated Plasmonic Nano-Pyramids for Bulk Heterojunction Solar Cell Photocurrent Enhancement, Nanoscale 4, 4421 (2012).

[46] J. Henzie, J. Lee, M. H. Lee, W. Hasan, and T. W. Odom, Nanofabrication of Plasmonic Structures, Annu. Rev. Phys. Chem. 60, 147 (2009).

[47] S. A. Maier, Plasmonics: Fundamentals and Applications (Springer, Berlin, 2007).

[48] A. Ordal, R. J. Bell, R. A. Alexander, Jr., L. L. Long, and M. R. Querry, Optical Properties of Fourteen Metals in the Infrared and Far Infrared: $\mathrm{Al}, \mathrm{Co}, \mathrm{Cu}, \mathrm{Au}, \mathrm{Fe}, \mathrm{Pb}, \mathrm{Mo}, \mathrm{Ni}$, Pd, Pt, Ag, Ti, V, and W, Appl. Opt. 24, 4493 (1985).

[49] E. Hao and G. C. Schatz, Electromagnetic Fields around Silver Nanoparticles and Dimers, J. Chem. Phys. 120, 357 (2004).

[50] A. B. Evlyukhin, C. Reinhardt, and B. N. Chichkov, Multipole Light Scattering by Nonspherical Nanoparticles in the Discrete Dipole Approximation, Phys. Rev. B 84, 235429 (2011).

[51] A. B. Evlyukhin, C. Reinhardt, E. Evlyukhin, and B. N. Chichkov, Multipole Analysis of Light Scattering by Arbitrary-Shaped Nanoparticles on a Plane Surface, J. Opt. Soc. Am. B 30, 2589 (2013).

[52] K. Kelly, E. Coronado, L. Zhao, and G. Schatz, The Optical Properties of Metal Nanoparticles: The Influence of Size, Shape, and Dielectric Environment, J. Phys. Chem. B 107, 668 (2003).

[53] W. Ji, K. Yao, and Y. C. Liang, Evidence of Bulk Photovoltaic Effect and Large Tensor Coefficient in Ferroelectric $\mathrm{BiFeO}_{3}$ Thin Films, Phys. Rev. B 84, 094115 (2011).
[54] V. Belinicher and B. Sturman, The Photogalvanic Effect in Media Lacking a Center of Symmetry, Sov. Phys. Usp. 23, 199 (1980).

[55] G. Chanussot and A. M. Glass, A Bulk Photovoltaic Effect due to Electron-Phonon Coupling in Polar Crystals, Phys. Lett. 59A, 405 (1976).

[56] S. M. Young and A. M. Rappe, First Principles Calculation of the Shift Current Photovoltaic Effect in Ferroelectrics, Phys. Rev. Lett. 109, 116601 (2012).

[57] A. Grachev, E. Nippolainen, and A. Kamshilin, Photogalvanic Effect Induced by an Electric Field, New J. Phys. 8, 78 (2006).

[58] V. I. Fal'ko and D. E. Khmel'nitskii, Mesoscopic Photovoltaic Effect in Microjunctions, J. Exp. Theor. Phys. 68, 186 (1989).

[59] J. Liu, M. A. Pennington, and N. Giordano, Mesoscopic Photovoltaic Effect, Phys. Rev. B 45, 1267 (1992).

[60] F. C. Champion, University Physics, Part 5 (Blackie \& Sons Ltd., Chicago, 1960), p. 728.

[61] V. V. Afanas'ev, Internal Photoemission Spectroscopy: Principles and Applications (Elsevier, Oxford, 2008).

[62] P. Reimann, Brownian Motors: Noisy Transport Far from Equilibrium, Phys. Rep. 361, 57 (2002).

[63] P. Hänggi, F. Marchesoni, and F. Nori, Brownian Motors, Ann. Phys. (Amsterdam) 14, 51 (2005).

[64] R. Corkish, M. A. Green, and T. Puzzer, Solar Energy Collection by Antennas, Solar Energy 73, 395 (2002).

[65] S. Sassine, Y. Krupko, J. C. Portal, Z. D. Kvon, R. Murali, K.P. Martin, G. Hill, and A.D. Wieck, Experimental Investigation of the Ratchet Effect in a Two-Dimensional Electron System with Broken Spatial Inversion Symmetry, Phys. Rev. B 78, 045431 (2008).

[66] L. Ermann, A. D. Chepelianskii, and D. L. Shepelyansky, Symmetry Breaking for Ratchet Transport in the Presence of Interactions and a Magnetic Field, Phys. Rev. E 87, 022912 (2013).

[67] V. K. Valev, N. Smisdom, A. V. Silhanek, B. De Clercq, W. Gillijns, M. Ameloot, V. V. Moshchalkov, and T. Verbiest, Plasmonic Ratchet Wheels: Switching Circular Dichroism by Arranging Chiral Nanostructures, Nano Lett. 9, 3945 (2009).

[68] T. Hatano, T. Ishihara, S. G. Tikhodeev, and N. A. Gippius, Transverse Photovoltage Induced by Circularly Polarized Light, Phys. Rev. Lett. 103, 103906 (2009). 